\title{
Photo-acoustic detection of chirality in metal-polystyrene metasurfaces
}

\author{
E. Petronijević, ${ }^{1, \text { a) }}$ G. Leahu, ${ }^{1}$ R. Li Voti, ${ }^{1}$ A. Belardini, ${ }^{1}$ C. Scian, ${ }^{2}$ N. Michieli, ${ }^{2}$ \\ T.Cesca, ${ }^{2}$ G.Mattei, ${ }^{2}$ C. Sibilia ${ }^{1}$ \\ ${ }^{1}$ Department S.B.A.I., Sapienza Università di Roma, Via A. Scarpa 14, I-00161 Rome, Italy \\ ${ }^{2}$ Physics and Astronomy Department, University of Padova, via Marzolo 8, I-35131 Padova, Italy
}

\begin{abstract}
Nowadays nanophotonics aims towards low-cost, chip-scale devices that can tailor electromagnetic properties, one of which is the control of the circular polarization at the nanoscale, important for novel optical devices. Here we show that nanosphere lithography, combined with tilted metal deposition, can provide novel metasurfaces with chiral properties. We apply the photo-acoustic technique to characterize the circular dichroism at $633 \mathrm{~nm}$ of polystyrene nanospheres covered by three different metals: Au- and Cr-covered samples show extrinsic chiral behavior, while the Ag-covered sample shows circular dichroism at normal incidence, characteristic for intrinsic chirality. As the experimental data are in good agreement with numerical predictions, we believe that such design can be optimized to get efficient circularly polarized detection at the nanoscale.
\end{abstract}

\section{THE MANUSCRIPT}

Chirality, a lack of the mirror symmetry of an object ${ }^{1}$, is an important property of some of the building blocks of our world: many molecules, amino-acids, DNA, sugars, drugs are chiral. Two mirror images of the same object differently interact with circularly polarized light of the opposite handedness, while having other measurable properties equal. In particular, chirality can affect the absorption and/or phase velocity of circularly polarized light, therefore it is possible to measure a difference in absorption directly related to the molecules' chirality. This measurement is known as Circular Dichroism (CD). At the nanoscale, when the nanostructures are comparable or smaller than the light wavelength, and organized periodically, they form a metasurface; generally, if the symmetry of the metasurface is broken, a chiral behavior is expected ${ }^{2}$. Chiral metasurfaces can manipulate electromagnetic fields and enhance the interaction with chiral molecules, important for chiral sensing ${ }^{3}$. On the other hand, they can control the polarization state of the optical field, or emit circularly polarized light, thus leading to applications in optical and quantum communications ${ }^{4}$. Geometric features of intrinsically chiral metasurfaces (the nanostructure in the unit cell is usually helix or gammadion-like) can be complicated to fabricate and implement at the nanoscale. This problem can be solved by a proper experimental set-up following the rule that the impinging light wavevector, the average surface normal, and the sample direction must be nonplanar. Such chiral behavior is called extrinsic chirality as it is governed by both experimental set-up and the

\footnotetext{
a) Electronic mail:emilija.petronijevic@uniroma1.it
} 
metasurface, which can be achiral itself ${ }^{5}$. Therefore, extrinsic chirality allows for the use of achiral nanostructures, which simplifies the nanofabrication, but provides CD only at oblique incidence $\mathrm{e}^{5-10}$.

Here we investigate the absorption properties of metasurfaces based on ordered polystyrene nanospheres (NS) monolayers, partially covered by metals ( $\mathrm{Au}, \mathrm{Cr}$, or $\mathrm{Ag}$ ). In such samples the combination of geometrical parameters (such as NS diameter and direction of metal deposition) and the type of metal leads to intriguing chiral behavior. We use photoacoustic spectroscopy to characterize different absorption for right and left circular polarization (RCP and LCP), giving $\mathrm{CD}$ as a direct result. Since experimental results are in good agreement with numerical work, we believe that the combination of low-cost fabrication technique and tilted metal evaporation can lead to novel efficient chiral designs.

Gold, chromium and silver tilted semi-nanoshell arrays (SNSAs) were produced by nanosphere lithography (NSL) on soda-lime glass substrates ${ }^{11-13}$, as explained in detail in Supp. Info 1. 2D arrays of hexagonal unit cells with polystyrene (PS) nanospheres of diameter $\mathrm{D}_{\mathrm{p}} \sim 370 \mathrm{~nm}$ were covered with a $50 \mathrm{~nm}$ thick metallic layer of $\mathrm{Au}, \mathrm{Cr}$ or $\mathrm{Ag}$ by thermal evaporation with a tilt angle of $45^{\circ}$ with respect to the sample's surface; for Ag-SNSA an additional in-plane tilt was introduced. Due to the non-close packed nature of the 2D array, a metallic layer (in the following labelled as the metallic grid) was formed at the substrate surface following the geometric shadow of the PS nanospheres. As an example, in Fig. 1(a)-(c) we report the FE-SEM images in plane view of the tilted Au-SNSA, Cr-SNSA, and Ag-SNSA samples, respectively, while a sketch of the samples is given in Fig. 1(d). For Ag-SNSA a more regular and prominent metallic grid was formed on the substrate, which is tilted with respect to the hexagon axes, leading to a specific asymmetry of this sample; the grid is thinner and less regular for the other two samples, thus introducing a negligible effect.
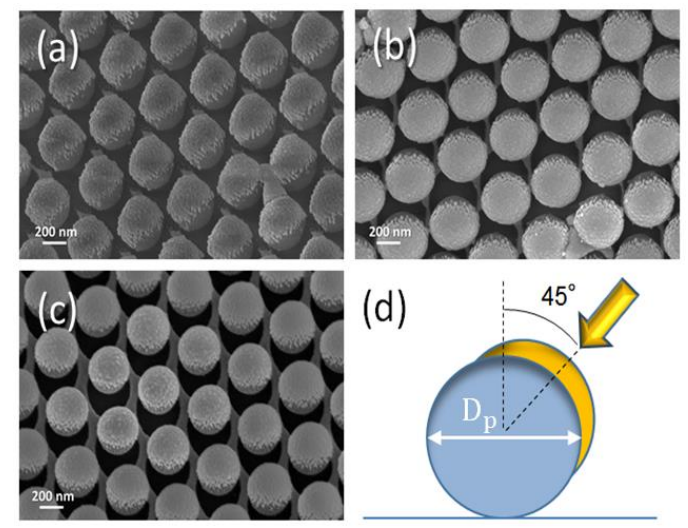

FIG. 1. Plane view FE-SEM images of the tilted (a) Au-SNSA, (b) Cr-SNSA, and (c) Ag-SNSA. (d) Sketch of the geometric parameters and the evaporation angle.

We then spectrally characterize our samples by measuring their absorption by means of the photo-acoustic (PA) technique ${ }^{14-17}$; the basic PA principles and the PA set-up details are given in Supp. Info 2. First, in order to distinguish the contribution of the nanostructure to the PA signal, we measure the spectra of an internal reference sample, which is a flat metallic film deposited on the same substrate near the 2D array of polystyrene NS during the evaporation, and normalize 
the results to the absorption of graphite, obtaining the spectra reported in Fig. 2(a). In the UV region they are all dominated by the absorption of the soda-lime glass substrate, in particular below $300 \mathrm{~nm}$. In the visible and near-infrared regions, the PA signal strongly decreases above the interband absorption threshold for $\mathrm{Au}$ and $\mathrm{Ag}$, while for $\mathrm{Cr}$ it decreases gradually; the soda-lime glass substrate shows negligible absorption. Next, we measure the PA spectra of SNSA arrays and normalize them to the zones without NS, Fig. 2(b). There is a clear absorption increase for Ag- and AuSNSA above the interband threshold wavelength, while for Cr-SNSA this ratio reaches 2. Such increase can be attributed to the fact that a continuous material dissipates heat better than a nanostructured one, especially if the nanostructure lies upon a scarce heat conductor such as polystyrene; this leads to a higher local temperature change for the SNSA, and, finally, to higher PA amplitude.

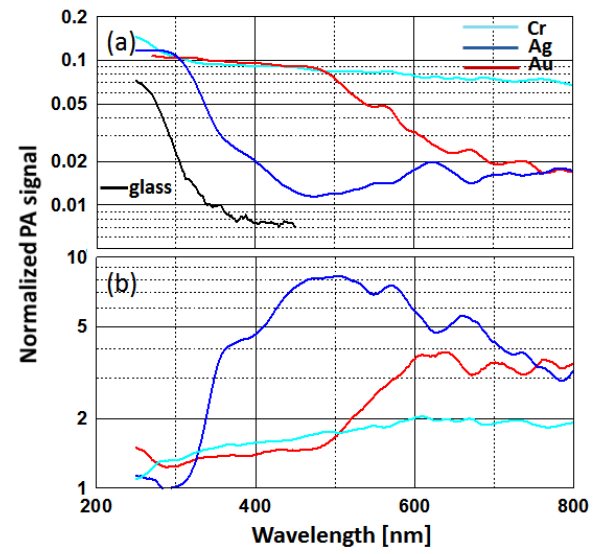

FIG. 2. (a) PA spectra of the soda-lime glass substrate (black) alone, and covered by a thin layer of Au (red), $\mathrm{Ag}$ (blue), or Cr (cyan); the spectra are normalized to the PA signal of graphite. (b) PA signal of SNSAs normalized to the respective flat metal film from Fig 2(a) for Au-SNSA (red), Ag-SNSA (blue), and Cr-SNSA (cyan).

Since all three samples have a notable PA increase at $633 \mathrm{~nm}$, we further chose this wavelength for CD characterization. We adapted the PA set-up for extrinsic chirality measurements, where we shine a $633 \mathrm{~nm}$ laser on the samples under an angle of incidence $\theta$ with respect to the sample normal $\vec{s}$. Since the metal deposition on top of the nanospheres is not symmetrical (thermal evaporation at $45^{\circ}$ ), with oblique incidence these structures are expected to exhibit extrinsic chirality under proper configuration, i.e., when the incidence wavevector $\vec{k}$, the average surface normal to the metallic nanocrescents $\vec{n}$ and the sample normal $\vec{s}$ are out of plane $e^{5,10,18}$ i.e $\vec{k} \cdot \vec{n} \times \vec{s} \neq 0$. A sketch of such experimental configuration is shown in Fig. S3(a): the substrate normal $\vec{s}$ lies parallel to the z-axis, while the average surface normal $\vec{n}$ is given by the evaporation direction. For Au and $\mathrm{Cr}$ samples, $\vec{n}$ and $\vec{s}$ lie in the $y z$-plane and form an angle of $45^{\circ}$ (Fig. S3(b)), while $\vec{k}$ and $\vec{s}$ lie in the $x z$-plane and form an angle $\theta$; the top view sketch of the structure is shown in Fig. S3(c). For the Ag sample, the projection of $\vec{n}$ on the $x y$-plane forms an angle $\varphi$ with respect to the y-axis (Fig. S4(b)).Therefore, we changed our PA set-up in order to be able to scan polarization and incidence angles (Fig. 3(a)). The laser light is linearly polarized before the quarter wave plate, so that it can be $\boldsymbol{s}$ or $\boldsymbol{p}$-polarized with respect to the metal flux direction when the wave plate's fast axis is at $0^{\circ}$, inset of Fig. 3(a). The wave plate fast axis scans the 
angles from $-180^{\circ}$ to $180^{\circ}$, where $-45^{\circ}$ represents $\mathrm{RCP}$ and $+45^{\circ}$ represents $\mathrm{LCP}$. The PA cell is mounted on a rotating stage, which enables the incidence angle scan from $\theta=-50^{\circ}$ to $50^{\circ}$. In Fig. 3(b) we show the PA signal dependence on the quarter waveplate rotation at $\theta=18^{\circ}$ for Au-SNSA; for comparison, we show the results obtained for the same sample before the Au evaporation. As expected, the structure without metal is completely symmetric and shows no difference for LCP and RCP absorption, while we note a different response at $\pm 45^{\circ}$ rotation when it is covered by Au. This is a typical behavior for extrinsically chiral nanostructures, which possess CD at oblique incidence ${ }^{9}$ We further introduce a figure of merit for $\mathrm{CD}$ in the PA measurements as:

$$
C D[\%]=\frac{A_{L C P}-A_{R C P}}{A_{L C P}+A_{R C P}} \cdot 200,
$$

where $A_{L C P}$ and $A_{R C P}$ are PA signal amplitudes for the quarter waveplate rotation of $+45^{\circ}$, and $-45^{\circ}$, respectively. For all the samples we observe CD only for the rotation of the sample around the projection of the metal flux ( $x z$-plane in Fig. S3(a)). In Fig. 3(c) we show the measured CD as a function of the incident angle for the three samples. We see that for the $\mathrm{Au}$ and $\mathrm{Cr}$ samples $\mathrm{CD}$ increases from $0 \%$ at normal incidence for positive angles of incidence, and becomes negative for negative angles, inverting its sign, which is typical for extrinsic chirality. The maximum CD is reached for Au-SNSA (CD of around $18 \%$ ) at $\theta \approx 25^{\circ}$, while a different behavior is observed for Cr-SNSA, which in this wavelength range has the maximum absorption, hence its geometric parameters play a minor role for CD. The most interesting is the Ag-SNSA whose $\mathrm{CD}$ varies rapidly as a function of the rotation angle from $-10^{\circ}$ up to $10^{\circ}$. In addition, at normal incidence this sample provides non-zero $\mathrm{CD}(\approx 7 \%)$, which is a property of intrinsically chiral structures ${ }^{19}$. Moreover, the overall absorption has a much higher contribution of the Ag grid on the substrate surface, formed due to the NS shadow during the evaporation, as it was recently reported in the plasmonic metasurfaces fabricated by a similar procedure ${ }^{13,20-22}$. However, the novelty of our metasurfaces lies in the tilted evaporation, in particular when nanocrescents and grids are rotated with respect to the hexagonal symmetry, as in the Ag sample: if the $x y$-plane projection of the flux direction $\vec{n}_{x y}$ lies parallel to one of the hexagonal symmetry directions, only extrinsic chirality can be observed under oblique angles, Fig. S4(a); otherwise, the offset angle between these directions makes the metallic grid and nanocrescents slightly rotated and asymmetric even at the normal incidence ${ }^{23}$, Fig. S4(b). 
(a)
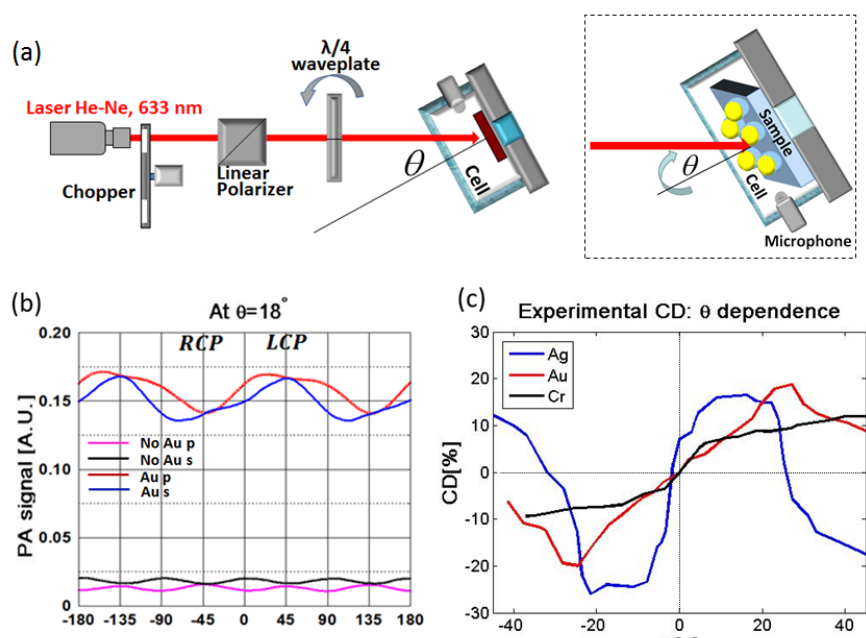

(c) Experimental CD: $\theta$ dependence

quarter waveplate angle $\left[{ }^{\circ}\right]$

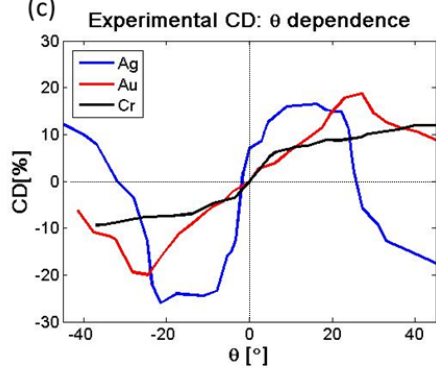

FIG. 3. (a) PA set-up for the chiral characterization: He-Ne laser is followed by a chopper and a linear polarizer, after which a quarter waveplate defines circular polarization. Inset: Sample with metallic cap under oblique incidence. (b) Quarter waveplate polarization scan at $\Theta=18^{\circ}$ of incidence, for PS nanospheres before and after the Au deposition. (c) CD dependence on the angle of incidence for the three samples.

In order to gain insights into the absorption properties at the points of interest, we modelled SNSA electromagnetic behavior in Lumerical ${ }^{24}$; the details are given in Supp. Info 3. In Fig. 4(a) the calculated CD is in very good agreement with PA results both in terms of shape and absolute values. Next, in Fig. 4(b) we show the absorption density calculated over the unit cell volume for the condition of maximum CD for Au-SNSA at $25^{\circ}$. As expected, the maximum absorption density is on the borders of the Au nanocrescent and controls the whole response of the metallic nanostructure; the grid on the substrate has negligible influence, and the absorption distribution at normal incidence is antisymmetric with respect to y-axis and equal when integrated over space for LCP and RCP (see 3D map in Fig. S6). In Fig. 4(c-e), for the sake of clarity, we show xy top view of the absorption maps. For Au-SNSA, at normal incidence, the antisymmetry of the distribution in Fig. 4(c) is in agreement with extrinsic chirality; consistently, at $\theta \approx 25^{\circ} \mathrm{LCP}$ is more absorbed than RCP, Fig. 4(d). In Fig. 4(e) we investigate Ag-SNSA at normal incidence: here LCP is more absorbed thus providing the positive non-zero $\mathrm{CD}$ due to the offset with respect to the hexagonal symmetry; as both the grid and the nanocrescents above the PS nanospheres are tilted providing the chiral behavior, at $\theta \approx 25^{\circ}$ they cancel out, thus leading to $C D=0$ (from the integration of the absorption in Fig. S7(b)). Finally, the removal of the nanospheres could lead to even higher CD of the grid which is made by elliptical nanoholes, as shown in CD simulation of the Ag nanogrid only in Fig. S8. This could make NSL technique of great interest for the chiral properties of surface plasmon polaritons as proposed in Ref. 25 , as it offers many degrees of freedom (periodicity, ellipse parameters, metal type, thickness, and tilting angle etc). 

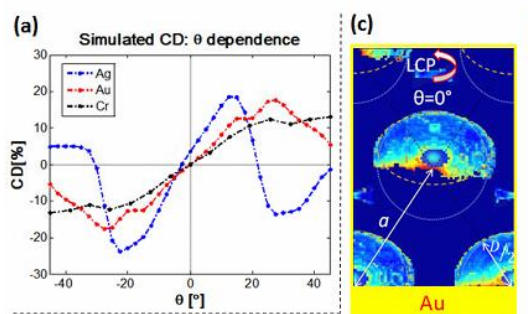

(d)

(e)
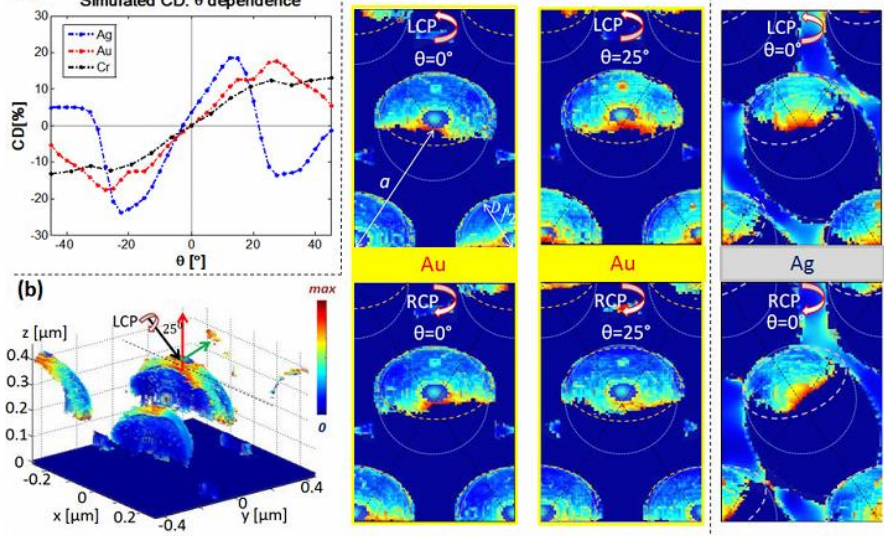

FIG. 4. (a) Calculated CD dependence on the angle of incidence. (b) 3D distribution of the absorption density in a unit cell of AuSNSA at $\theta=25^{\circ}$ and LCP (maximum CD). Top views (xy plane) of the absorption maps shown in Fig. S5-6 for opposite handednesses for (c) Au-SNSA at $\theta=0^{\circ}$, (d) Au-SNSA at $\theta=25^{\circ}$, and (e) Ag-SNSA at $\theta=0^{\circ}$.

In conclusion, in this work we have presented the $\mathrm{CD}$ behavior of metasurfaces consisting of polystyrene nanospheres asymmetrically covered with $\mathrm{Au}$, Ag or $\mathrm{Cr}$. Such semi-nanoshell arrays (SNSAs) were realized by nanosphere lithography (NSL) on soda-lime glass substrates and tilted evaporation of the metals. An extrinsic chiral response is detected as a $\mathrm{CD}$ dependence on the input beam angle of incidence. The maximum $\mathrm{CD}$ is observed at $25^{\circ}$ for Au-SNSA, the least pronounced CD is found for $\mathrm{Cr}$, while the Ag-covered sample offers novel behavior due to the tilted deposition with respect to the hexagonal symmetry, thus providing CD also at normal incidence. The numerical calculations closely follow PA results, and reveal that this geometry, together with the absorption properties of metals, can tailor $\mathrm{CD}$ and control field localization and distribution. Finally, we propose nanosphere lithography along with the tilted metal evaporation for the fabrication of novel chiral devices, whose chiral behavior can be characterized by scattering-free, stable and simple PAS technique. We do believe that such approach can lead to applications in both chiral sensing and chiral light manipulation at the nanoscale.

\section{ACKNOWLEDGMENTS}

Marco Magi, at S.B.A.I.,is kindly acknowledged for technical support.

\section{References}

${ }^{1}$ L. Kelvin, The Molecular Tactics of a Crystal (Clarendon Press, Oxford, 1894).

${ }^{2}$ T. Verbiest, M. Kauranen, A. Persoons, M. Ikonen, J. Kurkela, H. Lemmetyinen, J. Am. Chem. Soc. 116, 9203 (1994).

${ }^{3}$ X.-T. Kong, L.V. Besteiro, Z. Wang, A. O. Govorov, Adv. Mater. 2018, 1801790 (2018).

${ }^{4}$ M. I. Dyakonov, Spin Physics in Semiconductors (Springer International Publishing, 2017)

${ }^{5}$ E. Plum, X.-X. Liu, V. A. Fedotov, Y. Chen, D. P. Tsai, N. I. Zheludev, Phys. Rev. Lett. 102, 113902 (2009).

${ }^{6}$ A. Belardini, M. Centini, G. Leahu, D. C. Hooper, R. Li Voti, E. Fazio, J. W. Haus, A. Sarangan, V. K. Valev,

C. Sibilia, Sci. Rep. 6, 31796 (2016).

${ }^{7}$ I. De Leon, M. J. Horton, S. A. Schulz, J. Upham, P. Banzer, R. W. Boyd, Sci. Rep. 5, 13034 (2015).

${ }^{8}$ T. Cao, C. Wei, L. Mao, Y. Li, Sci. Rep. 4, 7442 (2014). 
${ }^{9}$ G. Leahu, E. Petronijevic, A. Belardini, M. Centini, C. Sibilia, T. Hakkarainen, E. Koivusalo, M. Rizzo Piton, S. Suomalainen, M. Guina, Adv. Opt. Mater. 5, 1601063 (2017).

${ }^{10}$ E. Petronijevic, M. Centini, A. Belardini, G. Leahu, T. Hakkarainen, C. Sibilia, Opt. Express 25(13), 17841 17848 (2017).

${ }^{11}$ T. Cesca, N. Michieli, B. Kalinic, I. G. Balasa, R. Rangel-Rojo, J. A. Reyes Esqueda, and G. Mattei, Mat. Sci. Semicon. Proc. (2018); DOI: 10.1016/j.mssp.2018.03.025.

${ }^{12}$ V. Russo, N. Michieli, T. Cesca, C. Scian, D. Silvestri, M. Morpurgo and G. Mattei, Nanoscale 9, 10117 (2017).

${ }^{13}$ T. Cesca, N. Michieli, B. Kalinic, A. Sanchez-Espinoza, M. Rattin, V. Russo, V. Mattarello, C. Scian, P. Mazzoldi and G. Mattei, Nanoscale 7, 12411-12418 (2015).

${ }^{14}$ G. Leahu, E. Petronijevic, A. Belardini, M. Centini, R. Li Voti, T. Hakkarainen, E. Koivusalo, M. Guina, C. Sibilia, Sci. Rep. 7, 2833 (2017).

${ }^{15}$ E. Petronijevic, G. Leahu, V. Mussi, C. Sibilia, and F. A. Bovino, AIP Adv. 7, 025210 (2017).

${ }^{16}$ E. Petronijevic, G. Leahu, A. Belardini, M. Centini, R. Li Voti, T. Hakkarainen, E. Koivusalo, M. Rizzo Piton, S. Suomalainen, M. Guina, C.Sibilia, Int. J. Thermophys. 39(3), 45 (2018).

${ }^{17}$ E. Petronijevic, G. Leahu, A. Belardini, M. Centini, R. Li Voti, T. Hakkarainen, E. Koivusalo, M. Rizzo Piton, S. Suomalainen, M. Guina, C.Sibilia, Int. J. Thermophys. 39(4), 46 (2018).

${ }^{18}$ A. Belardini, M. C. Larciprete, M. Centini, E. Fazio, C. Sibilia, D. Chiappe, C. Martella, A. Toma, M. Giordano, F. Buatier de Mongeot, Phys. Rev. Lett. 107, 257401 (2011).

${ }^{19}$ A.Belardini, A. Benedetti, M. Centini, G. Leahu, F. Mura, S. Sennato, C. Sibilia, V. Robbiano, M. C. Giordano, C. Martella, D. Comoretto, F. Buatier de Mongeot, Adv. Opt. Mater. 2, 208-213 (2014).

${ }^{20}$ H. Sánchez-Esquivel, K. Y. Raygoza-Sánchez, R. Rangel-Rojo, E. Gemo, N. Michieli, B. Kalinic, J. A. ReyesEsqueda, T. Cesca, G. Mattei, Sci. Rep. 7, 5307 (2017).

${ }^{21}$ Z. Li, R. E, C. Lu, A. Bimo Prakoso, M. Foldyna, R. Khoury, P. Bulkin, J. Wang, W. Chen, E. Johnson, P. Cabarrocas Caoa, Nanomaterials 8(8), 626 (2018).

${ }^{22}$ A. Benedetti, A. Belardini, A. Veroli, M. Centini, C. Sibilia, J. Appl. Phys. 116, 164312 (2014).

${ }^{23}$ V. E. Bochenkov, D. S. Sutherland, Opt. Express 26(21), 27101-27108 (2018).

${ }^{24}$ Lumerical Solutions, Inc. http://www.lumerical.com/tcad-products/fdtd/

${ }^{25}$ T. Cao, M. J. Cryan, J Electromagnet Wave and Applications 26 (10) 1275-1282 (2012).

\section{Supporting Information}

\section{Fabrication methods}

Gold, chromium and silver tilted semi-nanoshell arrays (SNSAs) were produced following a three-step process. First, commercial polystyrene (PS) nanospheres (MicroParticles GmbH, Germany) of $522 \mathrm{~nm}$ diameter were self-assembled to form a close-packed monolayer on the soda-lime glass substrates14. Then, the PS nanospheres were etched by Reactive Ion Etching (RIE) in an Ar-O2 atmosphere (30\% $\mathrm{Ar}-70 \% \mathrm{O}_{2}$ ) at $15 \mathrm{~W}$ for $10 \mathrm{~min}$ to reduce their diameter (to about $\mathrm{Dp}=370 \mathrm{~nm}$ ), preserving the $2 \mathrm{D}$ ordered arrangement. As a last step, a $50 \mathrm{~nm}$ thick metallic layer of $\mathrm{Au}, \mathrm{Cr}$ or $\mathrm{Ag}$ was deposited on the PS nanospheres by thermal evaporation with a tilt angle of $45^{\circ}$ with respect to the sample's surface; for Ag-SNSA an additional in-plane tilt was introduced. A calibrated quartz microbalance was used to control the thickness of the deposited layers during the evaporation.

\section{Photo-acoustic technique}

PA is a photothermal technique in which absorption of an incoming light beam leads to a non-radiative deexcitation process that generates heat. If the intensity of the light is modulated in time, a sample in an air-tight chamber heats up and cools down in a cycle and, not having time to expand and contract, corresponding changes in pressure arise. These pressure changes produce an acoustic signal which is directly converted into an electrical one by a sensitive micro-phone. The microphone receives the signal from the sample through the small diameter tunnel (labyrinth) so that light cannot impinge the microphone, thus largely reducing the effects of the scattered light. This way we can directly measure local absorption, and possibly detect the effects that would be otherwise hidden with other scattering dependent techniques.

Spectral PA measurement were done at normal incidence by a white light Xe arc source followed by a monochromator, which provides a spectral range from $250 \mathrm{~nm}$ to $1100 \mathrm{~nm}$, Fig. S2. Here, the source is modulated by a mechanical chopper, whose modulation frequency has been set at $81 \mathrm{~Hz}$ for all the measurements in this work. The output of the microphone pre-amplifier is connected to the input of the lock-in amplifier that analyses the signal with respect to the modulation frequency of the light source. The main element of the PA cell is a movable quartz cylinder, with two optically polished faces. The cylinder offers some advantages: its movement in $\mathrm{z}$-direction changes the distance between the sample surface and the window, so 
that we can choose the optimum volume of the cell to maximize the signal, while the transmitted light leaves the cell through it, thus reducing the noise.

\section{Simulation methods}

In order to investigate the absorption behavior of SNSAs at the nanoscale, we performed numerical simulations using a commercial-grade numerical package based on the 3D Finite Difference Time Domain (FDTD) method in Lumerical; the geometric parameters of the samples were determined from the fabrication parameters and the FE-SEM characterizations. The simulation domain is defined by a unit cell with periodic boundary conditions in $\mathrm{x}$ and $\mathrm{y}$ directions (Fig. S3(c)), and perfect matching layers in $\mathrm{z}$ direction. The absorption per unit volume is calculated as $\rho_{a b s}=-0.5 \omega^{2}|E|^{2} \operatorname{Im}\{\varepsilon\}$, by monitoring both electric field and complex refractive index across the simulation domain. Two plane waves with properly shifted phases are used to model the circular polarization, at the angles of incidence as in PA measurements. Metallic grids deposited on the substrate are modelled by subtracting the nanospheres' shadow from the metallic layer upon the soda-lime glass. This shadow is modelled as an ellipsoid with dimensions and position dependent on both $\vec{n}$ and $\vec{n}_{x y}$ (Fig. S4). Moreover, for Ag-SNSA both metallic nanocrescents upon the polystyrene NS and the grid are rotated by the offset angle $\phi$, in order to account for its peculiar intrinsically chiral behavior, Fig. S4(b). In Fig. S5 we show CD dependence on $\phi$; the intrinsic chiral behavior $\left(C D=0\right.$ at $\Theta=0^{\circ}$ and $\left.C D(\Theta)=-C D(-\Theta)\right)$ is noted at $\phi=0^{\circ}$ or $\phi=180^{\circ}$, while $C D$ is zero for all $\Theta$ at $\phi=90^{\circ}$ or $\phi=270^{\circ}$ as the vectors in Fig. S3(a) become co-planar. We find that the offset angle $\phi=20^{\circ}$ gives good fit to the experimental results.

\section{Supporting figures}

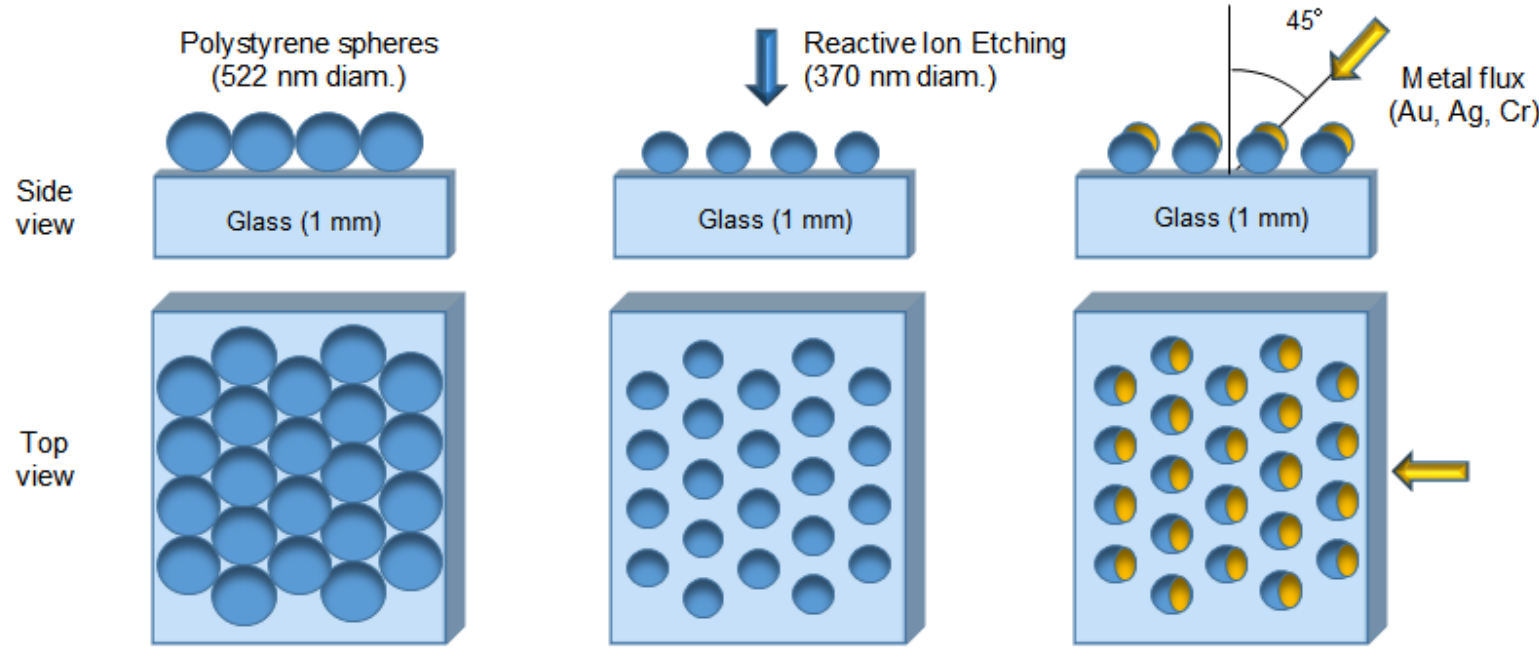

Fig. S1: Three steps of the fabrication process: (1) Polystyrene nanospheres (NS) are selfassembled in a monolayer on a soda-lime glass substrate; (2) Reactive Ion Etching of the nanospheres is done to reduce their diameter; (3) tilted $\left(45^{\circ}\right)$ thermal evaporation of the metals $(\mathrm{Au}, \mathrm{Ag}, \mathrm{Cr})$ is performed. 


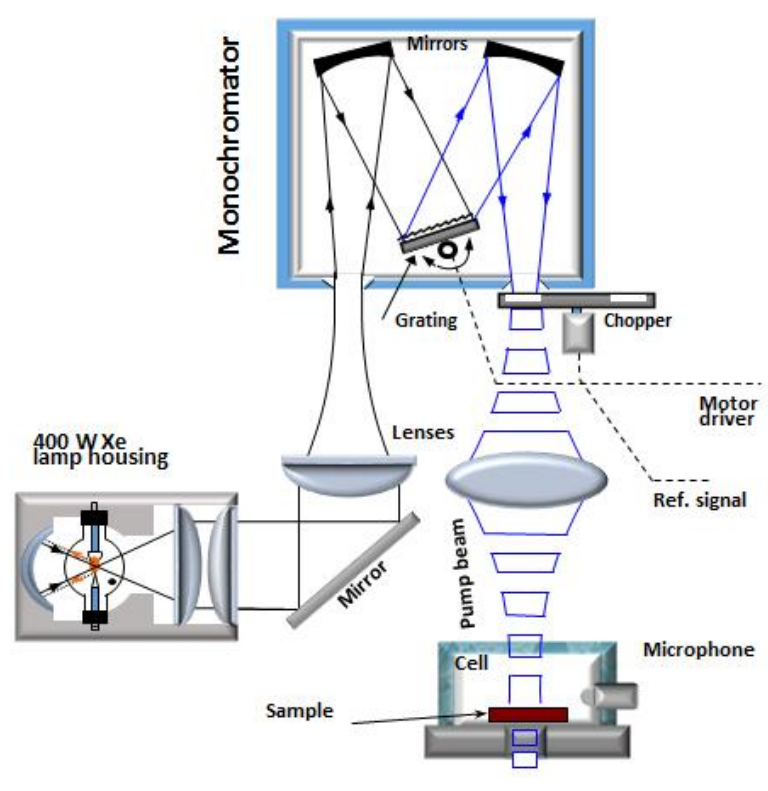

Fig. S2: Photo-acoustic spectroscopy set-up for the spectral characterization at normal incidence.
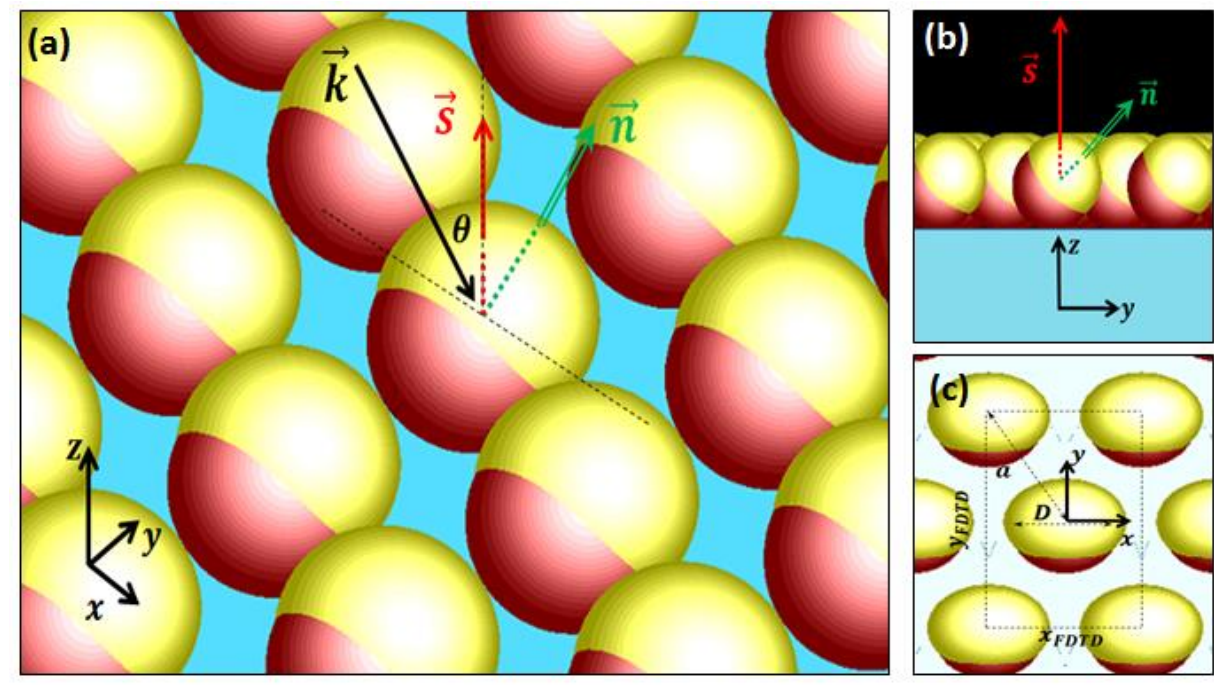

Fig. S3: (a) Close sketch of the extrinsic chirality measurement. CD is expected at oblique incidence $\left(\theta \neq 0^{\circ}\right)$ when the sample direction $\vec{s}$, the incident wave vector $\vec{k}$, and the average surface normal $\vec{n}$ do not lie in the same plane; (b) xz cross section of the sketch. (c) xy top view of the sketch. 

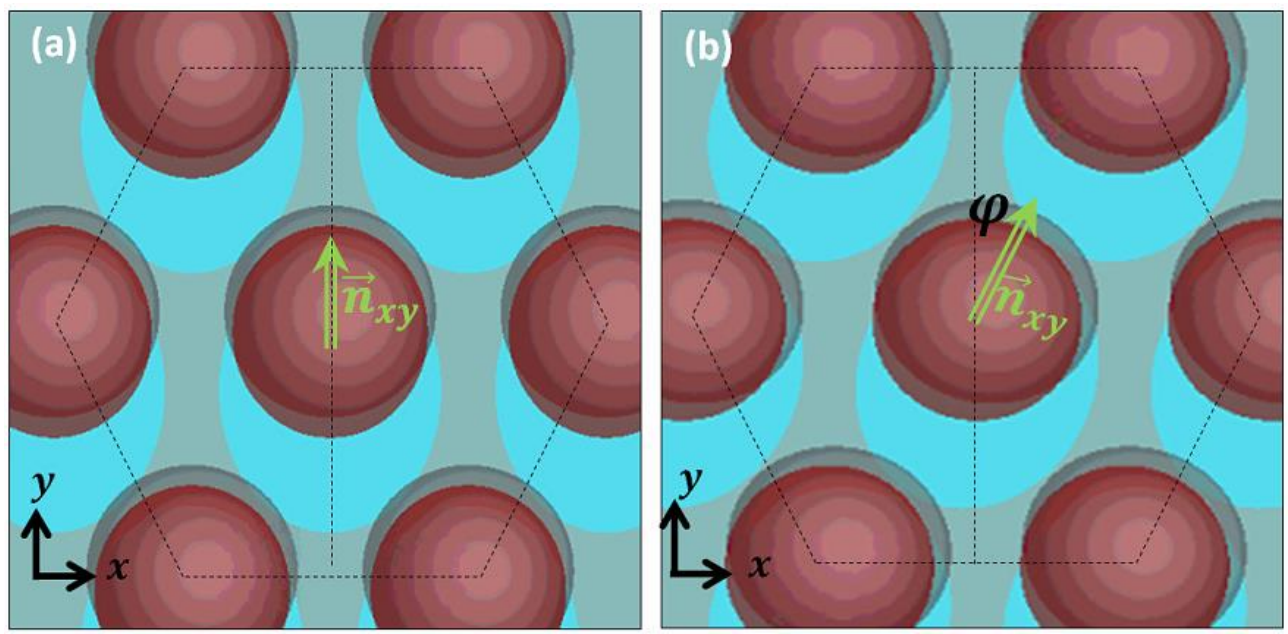

Fig. S4: Shadow and the metallic grid on the substrate when the tilted metal deposition projection on the xy plane is (a) parallel, and (b) not parallel to one of the hexagonal symmetry directions.

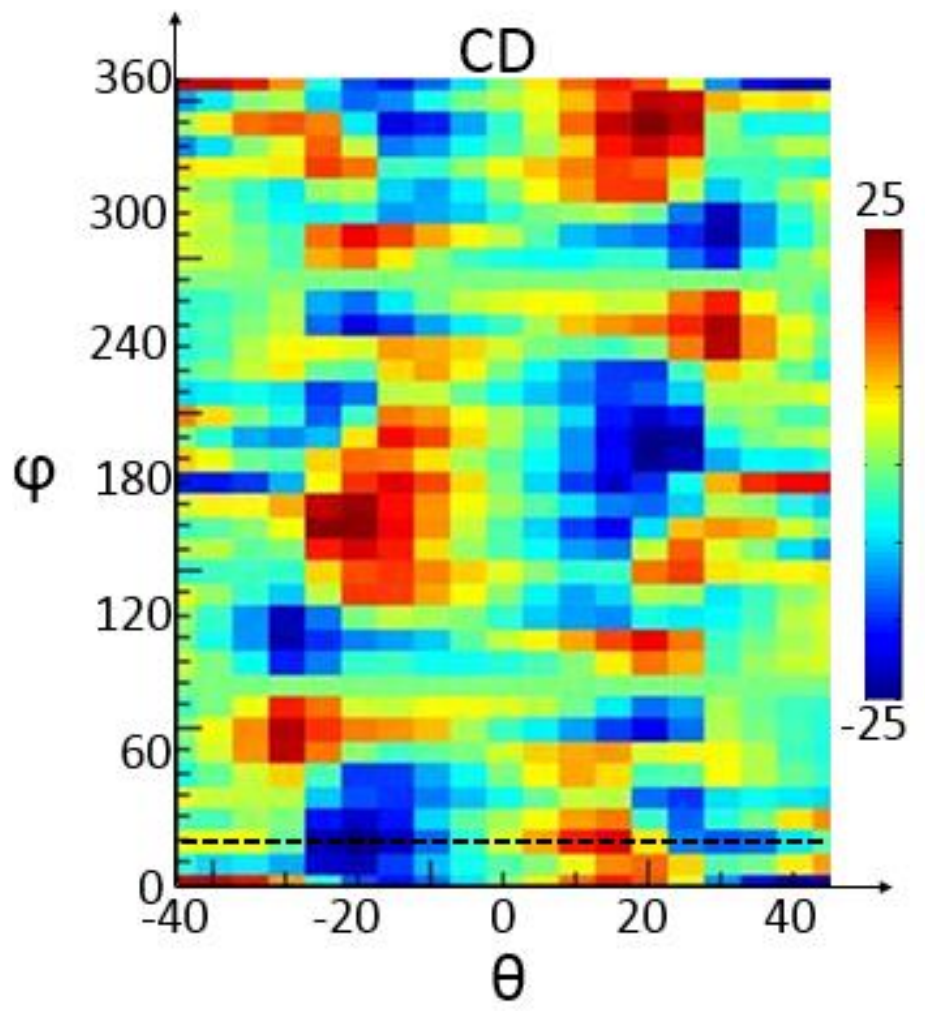

Fig. S5: Map of the Ag-SNSA CD as a function of the offset asymmetry angle $\varphi$. The dashed line shows the best fit position. 

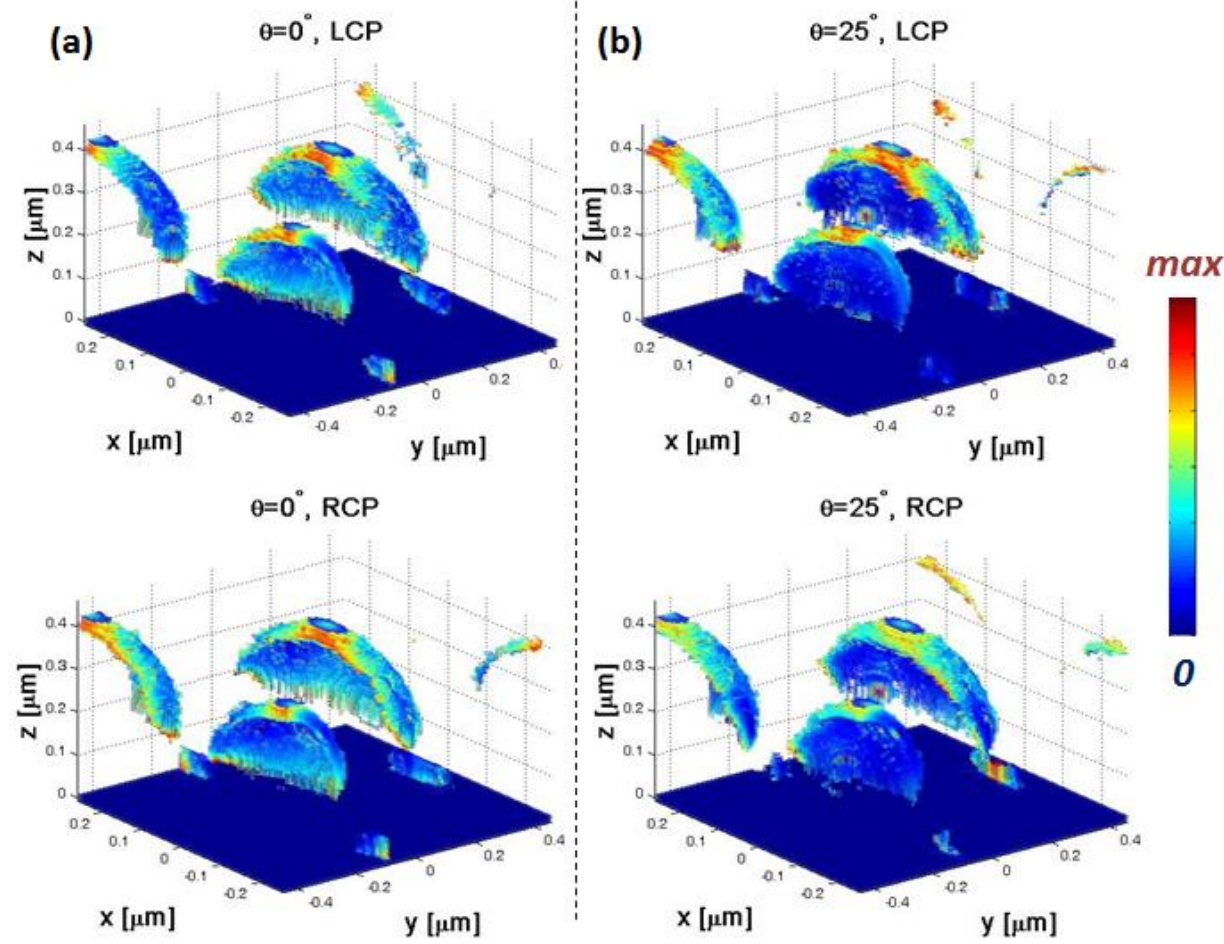

Fig. S6: 3D absorption distribution in Au-SNSA for the different handedness under (a) normal incidence; (b) $\theta=25^{\circ}$ (maximum CD).

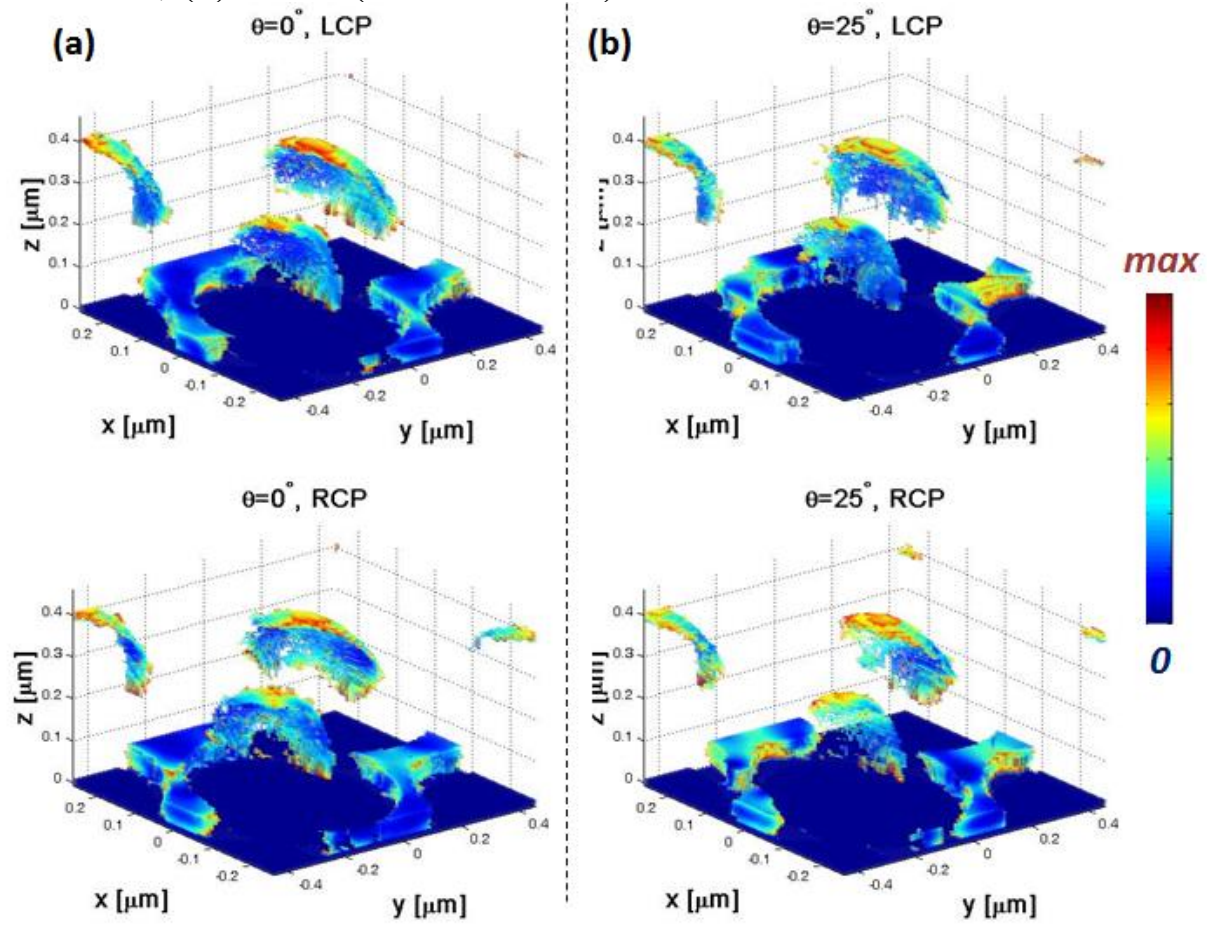

Fig. S7: 3D absorption distribution in Ag-SNSA for the different handedness under (a) normal incidence; (b) $\theta=25^{\circ}$ (zero CD). 


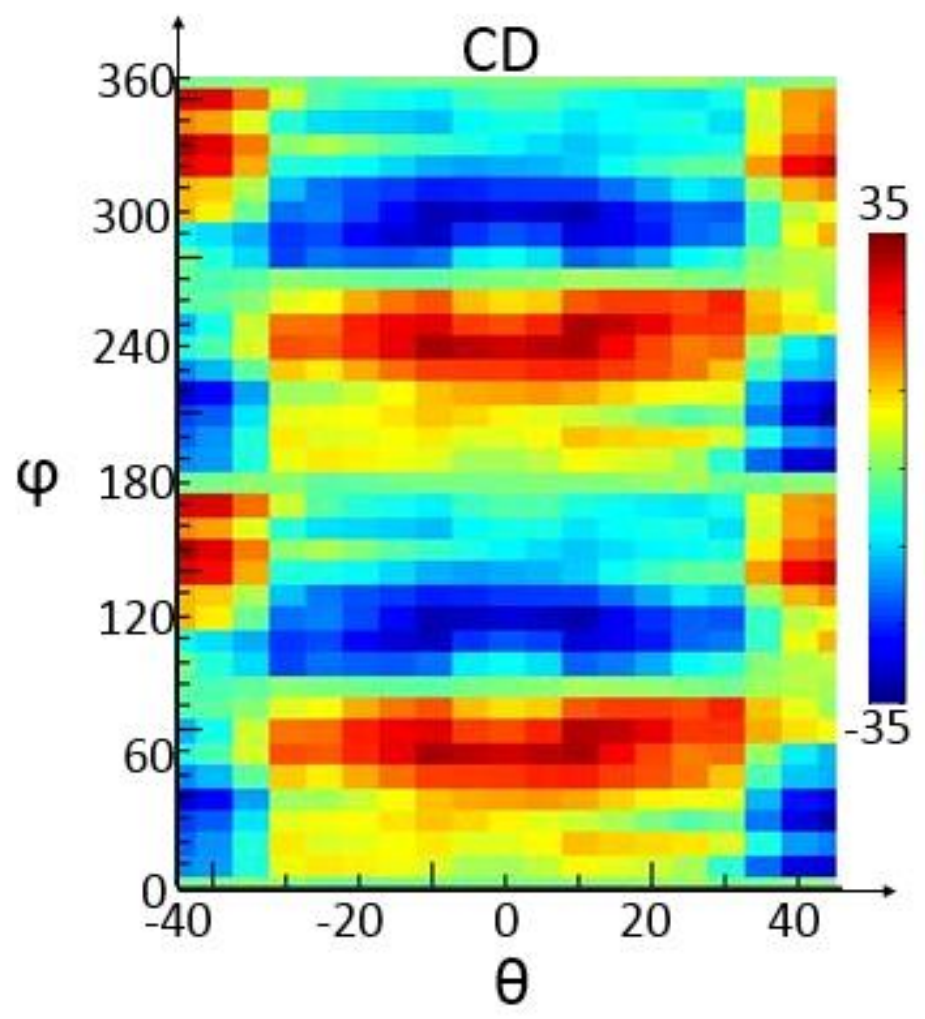

Fig. S8: CD dependence of the offset asymmetry angle $\varphi$ without the nanospheres, calculated for the Ag nanogrid of the sample Ag-SNSA. 